\title{
Secure and Privacy-Preserving Information Brokering
}

\author{
Peng Liu \\ Pennsylvania State University, USA
}

\begin{abstract}
In contrast with the situations when the information seeker knows where the needed data is located, a Distributed Information Brokering System (DIBS) needs to help each information seeking query "locate" the corresponding data source(s). Although DIBSs face daunting maintenance challenges, the data source locationing capability is highly desired in many important applications, such as emergence health care. Due to this new capability, existing anonymous communication techniques, such as Chaum Mix and Tor, are no longer sufficient. And the privacy enhancing measures must be integrated with the query routing operations.

In this work, we present a flexible and scalable DIBS using a brokercoordinator overlay network. Through a novel automaton segmentation scheme, in-network access control, and query segment encryption, our system integrates security enforcement and query forwarding while preserving system-wide privacy.
\end{abstract}

